\title{
Experimental and numerical analysis of directional added mass effects in partially liquid-filled horizontal pipes
}

\author{
Xavier Escaler ${ }^{\mathrm{a},}$, Oscar De La Torre ${ }^{\mathrm{b}}$, Jamie Goggins ${ }^{\mathrm{b}}$ \\ ${ }^{a}$ Centre for Industrial Diagnostics and Fluid Dynamics (CDIF), Universitat Politècnica de Catalunya, Av. Diagonal 647, 08028 Barcelona, Spain \\ ${ }^{\mathrm{b}}$ Centre for Marine Renewable Energy Ireland (MaREI), National University of Ireland Galway, University Road, Galway, Ireland
}

\section{A R T I CLE INFO}

\section{Keywords:}

Fluid-structure interaction

Liquid-filled pipe

Experimental modal analysis

FEM acoustic analysis

Added mass coefficient

\begin{abstract}
A B S T R A C T
The change of pipe natural frequencies due to added mass effects has been investigated in two cylindrical horizontal pipes from empty to completely water filled cases with various intermediate partially-filled conditions. The added mass coefficients of the three first vertical and horizontal modes of vibration have been determined with both experimental modal analysis and finite element analysis (FEA) acoustic-structural numerical simulations, which showed good agreement. The vertical and horizontal added mass coefficients present different behaviors as a function of the water level. Moreover, the pipe cross sectional dimensions determine the magnitude of these effects. For generalization to any pipe size, dependency of the directional added mass coefficients with new vertical and horizontal added mass estimators has been found. These estimators can be used in practical situations with horizontally mounted cylindrical pipes as a reference to predict and quantify air content.
\end{abstract}

\section{Introduction}

The interest in horizontal pipeline flow of air-water mixtures started many years ago, as shown by the experimental work of Govier and Omer (1962) in 1962 who compared various methods to correlate the pressure drop with the air-water ratio. As clearly exposed by Escarameia (2007), the presence of air in pipe systems can result in problems such as carrying capacity drop, flow disruption and reduced pump efficiency. Consequently, in large piping systems, considerable costs are incurred to remove the accumulated air due to dissolved gas or entrapment at pumps or air valves. In pressurized piping systems where optimal air release systems are too expensive or where the operation requirements do not permit to regularly apply hydraulic removal actions, a method for detection and diagnosis of gas pockets is of interest to avoid operation failure or undesirable discharge levels. For example, Lubbers and Clemens (2007) have carried out several experiments to detect gas problems using dynamic system response analysis. Such nondestructive methods are obviously helpful to overcome potential problems by applying remedial measures in advance before any unexpected catastrophic failure.

Pipe systems conveying fluid are Fluid-Structure Interaction (FSI) systems that have been modeled and simulated with different procedures, such as the ones proposed by Lavooij and Tijsseling (1991) for instance. A broad overview of the literature pertaining to the dynamic analysis of fluid-filled systems considering FSI are presented by Li et al. (2015).

In the present work, it is intended to investigate the suitability of detecting and quantifying the presence of gas pockets in circular cylindrical horizontal shells by assuming that their natural frequencies will change relative to the fully liquid-filled condition due to added mass effects. The presence of an incompressible, inviscid liquid partially filling a clamped-free pipe was theoretically, numerically and experimentally proved to change its modal properties as a function of liquid level by Chiba et al. (1984a, 1984b,

Corresponding author.

Email addresses: escaler@mf.upc.edu (X. Escaler); oscar.delatorre@nuigalway.ie (O. De La Torre); jamie.goggins@nuigalway.ie (J. Goggins) 
1985). Amabili and Dalpiaz (1995) and Amabili (1996, 1997a, 1999) from 1995 to 1999 carried out extensive theoretical and experimental studies to obtain exact solutions for the free vibration problems of circular cylindrical tanks and pipes from partially liquid-filled conditions to completely filled and partially immersed in dense fluids. As a result, the natural frequencies and the mode shapes were theoretically found as a function of the water level and validated through comparison with results of experimental modal analyses. A different numerical approach was proposed by Chen and Ding (1999), Mazúch et al. (1996) and Ergin and Temarel (2002), who used the finite element method (FEM). In particular, the latter authors calculated the FSI effects in terms of generalized added masses.

In particular, the natural frequencies of two horizontally free mounted pipes with nominal diameters of DN32 and DN50 (ISO) will be calculated as a function of the water filling level. Experimental modal analyses and numerical simulations results will be compared and validated. Then, frequency ratios and added mass coefficients will be analyzed and discussed for vertical and horizontal directions. And finally, two mathematical expressions to estimate the added mass effects as a function of water level will be proposed that can be used in practical situations to predict the presence of large air pockets in pipes.

\section{Theoretical approach}

Unlike simple structures such as beams, the mathematical approach to study the dynamic behavior of cylindrical shells is far more complex. Most of the published work by Amabili (1997b) and Amabili et al. (2001) focus on the simply supported case because this boundary condition applied to both ends of the shell greatly simplifies the computational effort. However, in the present work, the authors have focused on a free-free condition shell which has received much less attention but could be considered as an ideally isolated basic case.

In particular, Warburton (1965) applied the generalized approach to the free-free condition shell, which was also reproduced by Blevins (1979). For that, the characteristic equation in matrix form, A, of an empty cylindrical shell is expressed with Eqs. (1)-(4):

A

$$
=\left[\begin{array}{lcr}
\left(A_{j}^{2}+\frac{1}{2}(1+k)(1-v) i^{2} \alpha_{2}\right)-\alpha_{2} \Omega^{2} & A_{j}\left(-v i \alpha_{1}-\frac{1}{2}(1-v) i \alpha_{2}\right) & A_{j}\left(-v \alpha_{1}+k\left(-A_{j}^{2}+\frac{1}{2}(\right.\right. \\
A_{j}\left(-v i \alpha_{1}-\frac{1}{2}(1-v) i \alpha_{2}\right) & \left(i^{2}+\frac{1}{2}(1+3 k)(1-v) A_{j}^{2} \alpha_{2}\right)-\Omega^{2} & i+k i A_{j}^{2}\left(v \alpha_{1}+\frac{3}{2}(1-\right. \\
A_{j}\left(-v \alpha_{1}+k\left(-A_{j}^{2}+\frac{1}{2}(1-v) i^{2} \alpha_{2}\right)\right) & i+k i A_{j}^{2}\left(v \alpha_{1}+\frac{3}{2}(1-v) \alpha_{2}\right) & \left(1+k\left(A_{j}^{4}+\left(i^{2}-1\right)^{2}+2 v i^{2} A_{j}^{2} \alpha\right.\right.
\end{array}\right.
$$

$A_{j}=\gamma_{J} \frac{R_{s}}{L_{s}}$

$k=\frac{e^{2}}{12 R_{s}^{2}}$

$\Omega^{2}=\omega^{2} R_{s}^{2}\left(\frac{\rho\left(1-v^{2}\right)}{E}\right)$

Where $\gamma_{J}$ is the equivalent beam frequency parameter, $R_{s}$ is the shell radius, $L_{s}$ is the axial length, $e$ is the wall thickness, $E$ is the Young's modulus, $\nu$ is the Poisson's ratio, $\rho$ is the density, $i$ and $j$ are the number of circumferential waves and longitudinal half-waves, respectively, $\alpha_{1}$ and $\alpha_{2}$ are parameters that depend on the boundary conditions and $\omega$ is the circumferential natural frequency. So, the natural frequencies can be obtained by solving the eigenvalue equation which can be expressed with Eq. (5):

$\operatorname{det}(\mathbf{A})=0$

To consider the effect of a fluid on the natural frequencies of a fully filled simply supported cylindrical shell, Amabili et al. (2001) used the factor $\chi$ expressed in Eq. (6).

$\chi=\frac{1}{\rho} \frac{I_{i+1}\left(\frac{j \pi R_{s}}{L_{s}}\right)}{I_{i+1}^{\prime}\left(\frac{j \pi R_{s}}{L_{s}}\right)}\left(\rho+\frac{\rho_{f} L_{s}}{j \pi R_{s}}\right)$

Where $I_{i}$ is the modified Bessel function of order I and $I_{i}^{\prime}$ its derivative and $\rho_{f}$ is the fluid density.

Unfortunately, the use of $\chi$ in A does not solve the natural frequencies of the free-free fully filled shell. Moreover, partially filled cases are even more complex to study analytically. 
Consequently, the current work intends to propose alternative empirical correlations which could give reasonable approximations with very low calculation effort. Specifically, the authors will focus on the first three natural frequencies of "beam-like" bending mode shapes which, mathematically, can be expressed by Eq. (7):

$i=1 ; j=1, \quad 2, \quad 3$

The theoretical three first natural frequencies of the empty shell can be obtained by inserting Eq. (7) into A, which will be used as reference values throughout this work.

\section{Tested cases}

In Fig. 1, a cross section of the horizontal cylindrical pipe partially filled with water is outlined with its main dimensions, which are the internal radius of the pipe, $R$, the pipe wall thickness, $e$, and the central angle and height of the circular segment determined by the water, $\theta$ and $h$, respectively. In this case, the water free surface is parallel to the horizontal axis of the pipe.

Based on geometry, the area, $A_{w}$, of the circular segment defined by the water and its height, $h$, are given by Eqs. (8) and (9), respectively.

$A_{w}=\frac{R^{2}}{2}(\theta-\sin \theta)$

$h=R\left(1-\cos \frac{\theta}{2}\right)$

Two steel pipes with nominal diameters of DN32 and DN50 and the same length $L$ of $1 \mathrm{~m}$ have been tested, as shown in Fig. 2 . The inner and outer diameters of the two pipes are given in Table 1.

For the tests, the pipes were filled with different volumes of water and two caps were fitted at both ends to avoid any leakage. The mass of each single cap is $0.216 \mathrm{~kg}$ for DN32 and $0.425 \mathrm{~kg}$ for DN50. In Table 2, the water volume $V_{w}$, the corresponding cen-

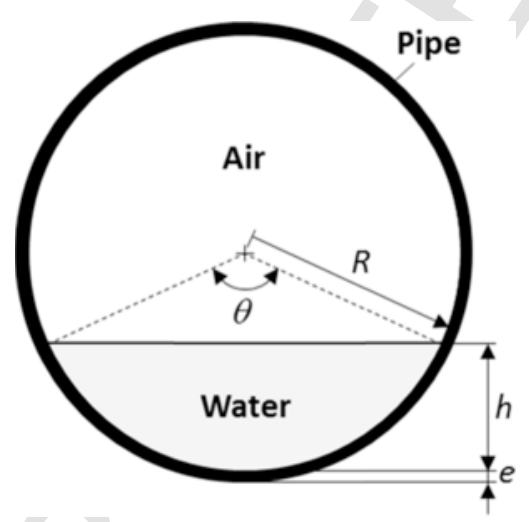

Fig. 1. Section and dimensions of the partially water-filled pipe.

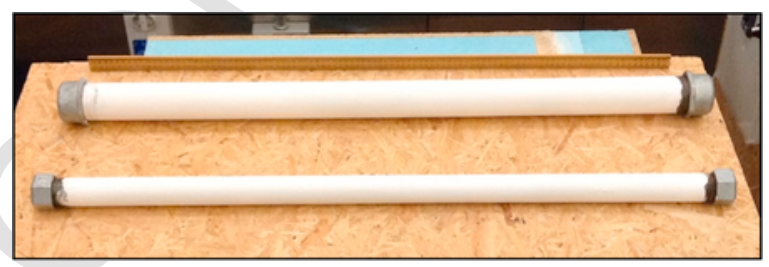

Fig. 2. DN32 and DN50 pipes.

Table 1

General dimensions of the pipes.

\begin{tabular}{lll}
\hline Pipe & DN32 & DN50 \\
\hline Outer diameter $[\mathrm{m}]$ & 0.04240 & 0.06030 \\
Inner diameter $[\mathrm{m}]$ & 0.03590 & 0.05300 \\
Wall thickness, $e[\mathrm{~m}]$ & 0.00325 & 0.00365 \\
Length, $L[\mathrm{~m}]$ & 1.0 & 1.0 \\
\hline
\end{tabular}


Table 2

Water volumes and corresponding dimensions of the circular segment.

\begin{tabular}{lllllll}
\hline Pipe & $V_{w}\left[\mathrm{~m}^{3}\right]$ & $\theta$ [deg. $]$ & $h[\mathrm{~m}]$ & Pipe & $V_{w}\left[\mathrm{~m}^{3}\right]$ \\
\hline DN32 & 0 & 0 & 0 & DN50 & 0 & 0 \\
DN32 & $200 \cdot 10^{-6}$ & 119.5 & 0.00891 & DN50 & $500 \cdot 10^{-6}$ & 0 \\
DN32 & $400 \cdot 10^{-6}$ & 159.5 & 0.01476 & DN50 & $1000 \cdot 10^{-6}$ & 125.5 \\
DN32 & $600 \cdot 10^{-6}$ & 194.5 & 0.02021 & DN50 & $1500 \cdot 10^{-6}$ \\
DN32 & $800 \cdot 10^{-6}$ & 233.0 & 0.02596 & DN50 & $2000 \cdot 10^{-6}$ & 0.01437 \\
DN32 & $1012 \cdot 10^{-6}$ & 360 & 0.03590 & DN50 & $2206 \cdot 10^{-6}$ \\
\hline
\end{tabular}

tral angle $\theta$ and the height $h$ are listed for each case with the cases of the pipes empty of water and completely full of water in the first and last rows respectively.

The experimental and numerical modal analysis of the pipes has been focused on the identification of the three first horizontal and vertical modes of vibration with 1,2 and 3 axial half-waves named as $f_{1}, f_{2}$ and $f_{3}$ respectively, as shown in Fig. 3.

\section{Experimental work}

This test has been carried out in the Structures Lab at the Civil Engineering Department in the National University of Ireland (Galway). Elastic ropes (0.01 m diameter) have been used to simulate free-free conditions for both pipes, as shown in Fig. 4 (left). These ropes were hung from an existing steel beam at $5 \mathrm{~m}$ height. This cantilever beam is part of a steel frame used to test heavy components and considered rigid. Hanging the pipes from such long ropes reduces the fundamental frequency and, hence, the risk of any undesirable coupling effect. Eq. (10) shows the simple pendulum frequency:
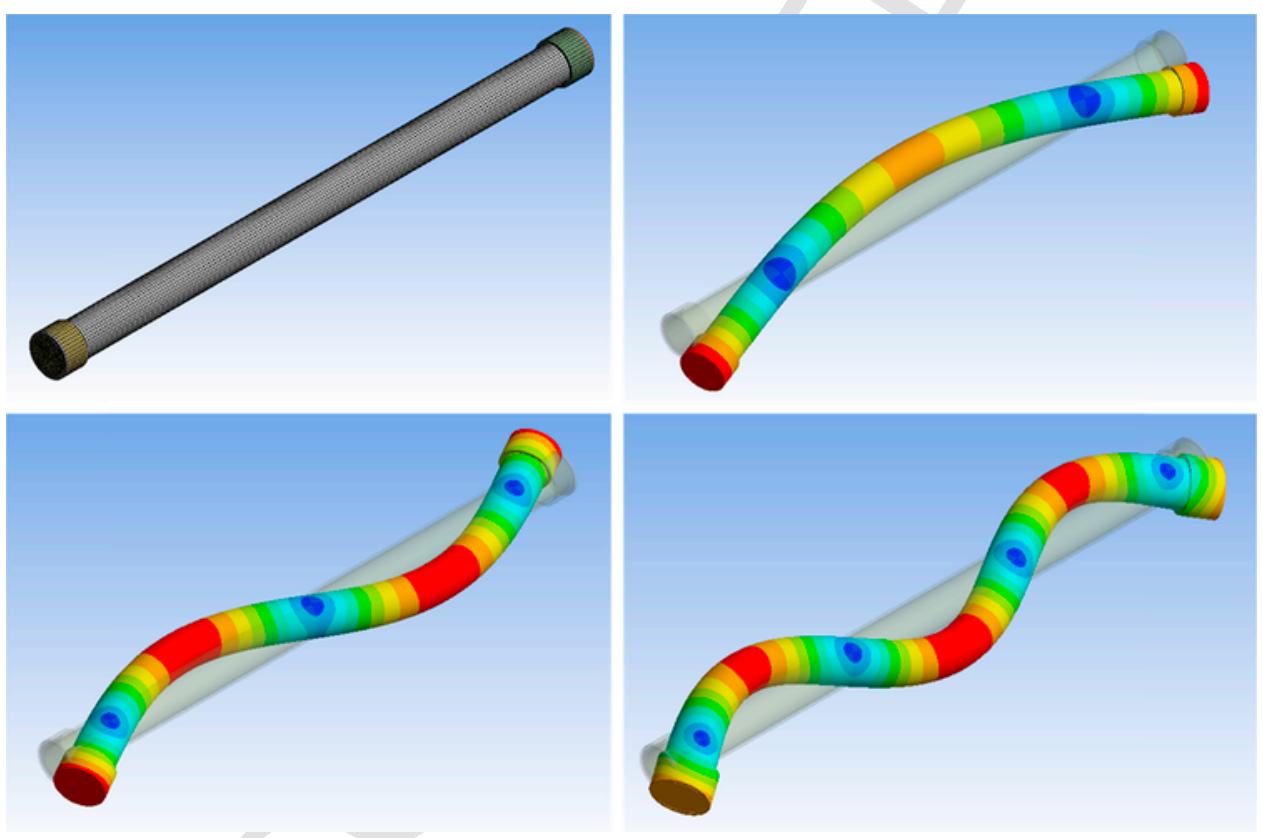

Fig. 3. FE mesh of the pipe (top left), first bending mode $f_{1}$ (top right), second bending mode $f_{2}$ (bottom left) and third bending mode $f_{3}$ (bottom right).
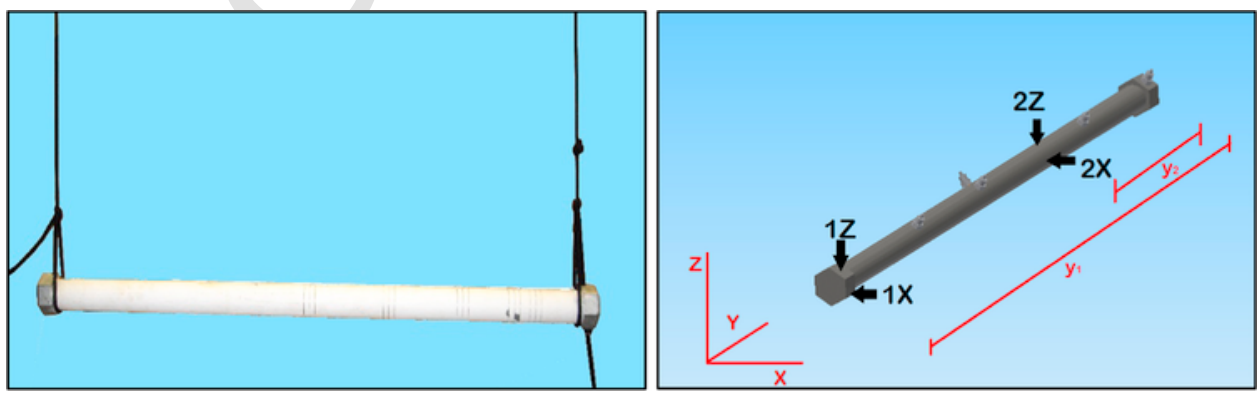

Fig. 4. DN32 hanging in the laboratory using the elastic ropes (left) and experimental setup scheme with the four different impact locations (right). 
$f_{0}=\sqrt{\frac{g}{L_{r}}}$

where $f_{0}$ is the frequency of the pendulum, $g$ is the gravity acceleration and $L_{r}$ the length of the rope.

\subsection{Test rig and acquisition system}

Five different accelerometers located along the pipe axis $Y$ have been used; four of them measuring in the vertical axis $Z$ and one of them in the horizontal axis $X$. Table 3 summarizes the accelerometers' data. Also an instrumented hammer, model Dytran M5802, has been used to excite both pipes.

The same approach has been adopted for both pipes: four different locations have been identified and marked on each pipe, two of them have been excited in $Z$ direction and the other two in $X$ direction, as seen in Fig. 4 (right). Impact points $1 Z$ and $1 X$ are located at approximately $\mathrm{y}_{1}=1 \mathrm{~m}$, whereas $2 \mathrm{Z}$ and $2 \mathrm{X}$ are located at $\mathrm{y}_{2}=0.31 \mathrm{~m}$.

The accelerometers were connected to a NI PXIe-4492 module within a PXI DAQ system. This 24-bit resolution module has 8 analog inputs simultaneously sampled and can acquire up to $204 \mathrm{kS} / \mathrm{s}$. Both pipes have been hit ten times on each impact location. 4500 samples at $5000 \mathrm{~S} / \mathrm{s}$ have been acquired for each hit. Frequency response functions (FRF) have been calculated for the different combinations (Single Input - Multiple Output) based on the averaged signals to obtain the system natural frequencies. In Fig. 5 some examples of the obtained FRF's are shown.

To obtain these FRF's, force-exponential windows have been applied to stimulus and response signals, respectively. The effective length of the response used to compute the FRF has been fixed so that its remaining level is $10 \%$ of the peak response as shown in Fig. 6.

Pipes have been filled with water by means of a pipette through one of the holes drilled to install an accelerometer. Once the desired water volume has been introduced in the pipe, the accelerometer has been reinstalled and the modal analysis performed. In each partial volume scenario, the peak identification has been performed.

\section{Numerical simulation}

\subsection{Acoustic-Structural FEA}

The coupled Acoustic-Structural analysis considers the structural dynamics equation, along with the linearized Navier-Stokes (NS) equations of flow momentum and continuity to solve the FSI problem. The NS equations are simplified to get the acoustic wave, shown in Eq. (11) in its discretized form, assuming that the fluid is compressible (density changes due to pressure variations) and that there is no mean flow (equal to zero).

$\mathbf{M}_{\mathbf{f}} \ddot{\mathbf{p}}+\mathbf{C}_{\mathbf{f}} \dot{\mathbf{p}}+\mathbf{K}_{\mathbf{f}} \mathbf{p}+\rho_{0} \mathbf{R}^{\mathrm{T}} \ddot{\mathbf{u}}=\mathbf{f}_{\mathbf{f}}$

Table 3

Specifications and mounting of the accelerometers.

\begin{tabular}{|c|c|c|c|c|}
\hline Accelerometer & Frequency range $[\mathrm{Hz}]$ & Location Y [m] & Axis alignment & Attachment type \\
\hline DYTRAN & $1-10000$ & 0.00 & $\mathrm{Z}$ & Magnet \\
\hline ENDEVCO 1888 & $1-8000$ & 0.21 & $\mathrm{Z}$ & Threaded \\
\hline ENDEVCO 1886 & $1-8000$ & 0.53 & $\mathrm{Z}$ & Threaded \\
\hline ENDEVCO 1887 & $1-8000$ & 0.53 & $\mathrm{X}$ & Threaded \\
\hline ENDEVCO 1885 & $1-8000$ & 0.73 & $\mathrm{Z}$ & Threaded \\
\hline
\end{tabular}

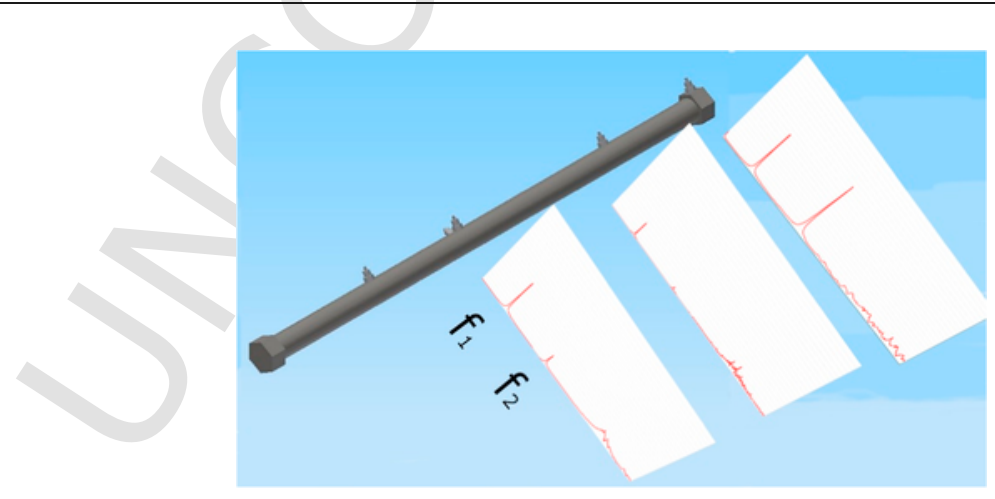

Fig. 5. Vertical FRF peaks for $f_{1}$ and $f_{2}$ at different accelerometer locations. 


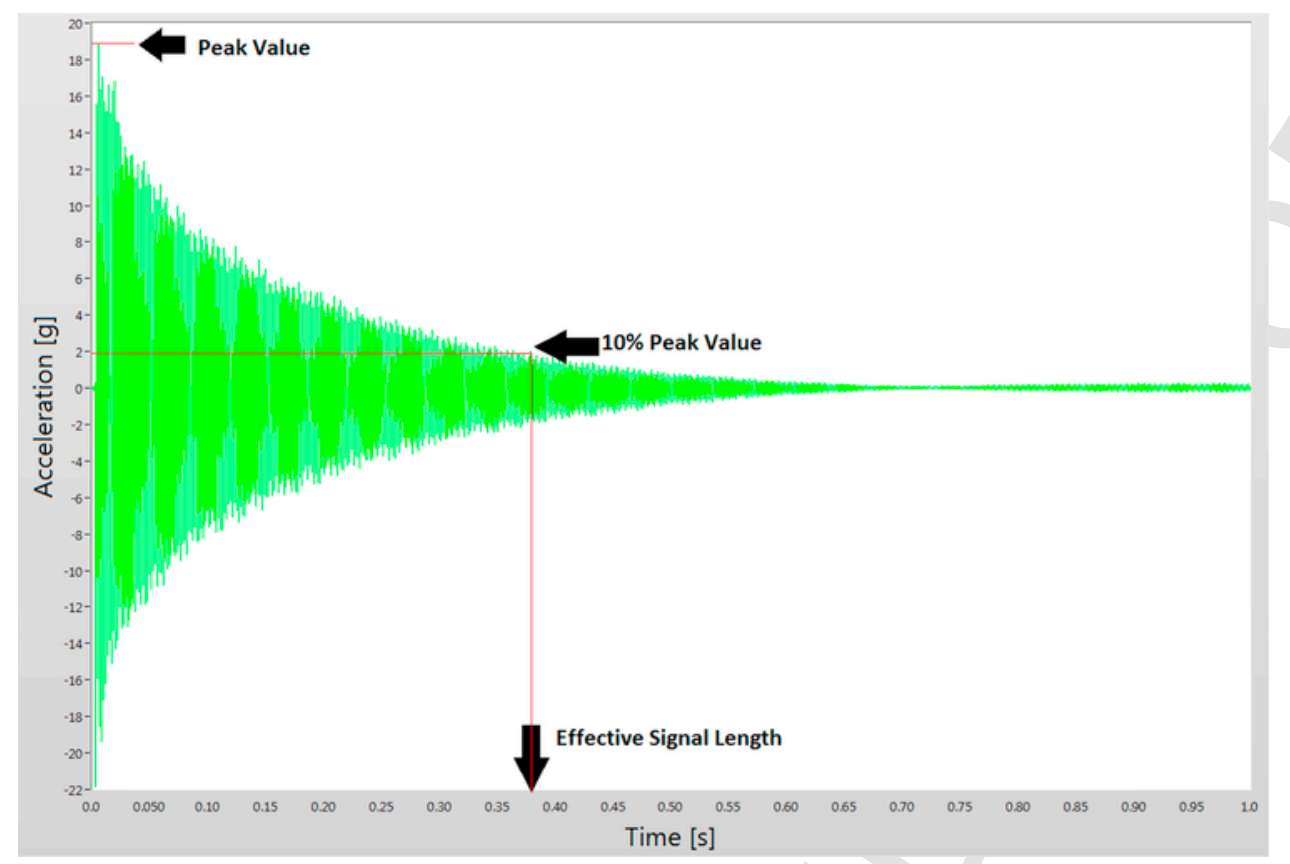

Fig. 6. Response signal after applying the exponential window.

where $\mathbf{M}_{\mathrm{f}}, \mathbf{C}_{\mathbf{f}}$ and $\mathbf{K}_{\mathbf{f}}$ are the acoustic fluid mass, fluid damping and fluid stiffness matrices, respectively, $\mathbf{R}$ is the acoustic fluid boundary matrix, $\mathbf{f}_{\mathbf{f}}$ is the acoustic fluid load vector, $\mathbf{p}$ is the acoustic pressure vector, $\mathbf{u}$ is the fluid element displacement vector and $\rho_{0}$ is the mean fluid density.

Consequently, pressure waves are generated in the fluid by the structure displacement and the structure deformation is affected by the pressure exerted by the fluid. The discretized structural and lossy wave equations are then combined to obtain the unsymmetrical coupled FSI matrix system, expressed in Eq. (12), so that they can be solved simultaneously.

$$
\left(-\omega^{2}\left[\begin{array}{ll}
\mathbf{M}_{\mathbf{s}} & 0 \\
\rho_{0} \mathbf{R}^{\mathbf{T}} & \mathbf{M}_{\mathbf{f}}
\end{array}\right]+j \omega\left[\begin{array}{ll}
\mathbf{C}_{\mathbf{s}} & 0 \\
0 & \mathbf{C}_{\mathbf{f}}
\end{array}\right]+\left[\begin{array}{ll}
\mathbf{K}_{\mathbf{s}} & 0 \\
0 & \mathbf{K}_{\mathbf{f}}
\end{array}\right]\right)\left\{\begin{array}{l}
\mathbf{u} \\
\mathbf{p}
\end{array}\right\}=0
$$

where $\omega$ is the angular frequency of the pressure oscillation, and $\mathbf{M}_{\mathbf{S}}, \mathbf{C}_{\mathbf{S}}$ and $\mathbf{K}_{\mathbf{S}}$ are the structure solid mass, damping and stiffness matrices, respectively.

\subsection{Model}

The model, created using ANSYS ${ }^{\circ}$ v. 14.5, comprises a structure domain for the pipe solid body and an acoustic fluid domain for the inner liquid and gas volumes filling the pipe as shown in Fig. 7. The material properties of the stainless steel, water and air considered in the calculation are listed in Table 4.
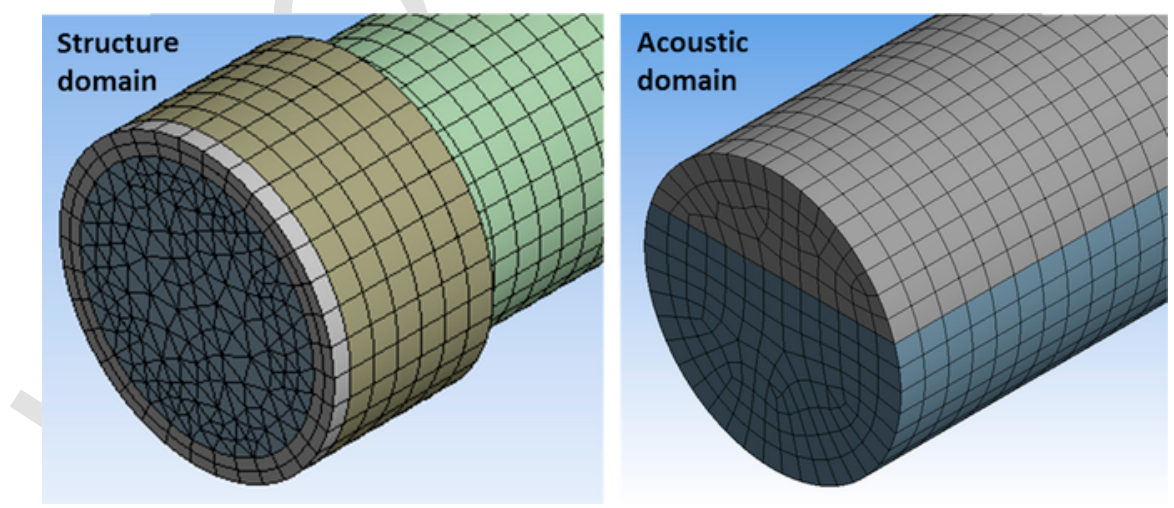

Fig. 7. Details of the structure solid domain (left) and the acoustic fluid domain with water at the bottom and air at the top (right). 
Table 4

Material properties considered in the numerical model.

\begin{tabular}{llll}
\hline Material & Density $\left[\mathrm{kg} / \mathrm{m}^{3}\right]$ & Sonic speed $[\mathrm{m} / \mathrm{s}]$ & Young Modulus $[$ Pa] \\
\hline Stainless steel & 7850 & - & $2 \cdot 10^{11}$ \\
Water & 998 & 1482 & - \\
Air & 1.2041 & 343.24 & - \\
\hline
\end{tabular}

As a result of the caps fitting, the effective length of the pipes is slightly increased. To account for this extra volume and mass, both FEM models are modified resulting in a total length of $1.03 \mathrm{~m}$ and $1.05 \mathrm{~m}$ for the DN32 and DN50 pipes, respectively.

A preliminary mesh sensitivity analysis has been carried out for both pipe sizes. Several meshes with total number of elements from 5000 to 73,000 and from 2000 to 137,000 for DN32 and DN50 pipes, respectively, present a maximum natural frequency deviation of about $0.2 \%$ for $f_{3}$ and lower deviations for $f_{2}$ and $f_{1}$. The final mesh refinement for both pipes, as shown in Figs. 3 and 8 , guarantees 2 radial divisions in the pipe wall, 250 axial divisions in both the pipe and the fluid, a minimum of 5 radial divisions in the fluid and an average element size of $0.005 \mathrm{~m}$. For the DN32 pipe the final meshes ranged from 43,000 to 50,000 elements. For the DN50 pipe the final meshes ranged from 35,000 to 60,000 elements. The mesh cross sections for each case are shown in Fig. 8.

For the acoustic domain, higher order solid elements with 20 nodes exhibiting quadratic pressure behavior were used. This element has four degrees of freedom per node including the translations in the nodal directions and the pressure. The percentage of fluid elements in the total domain was around 50\%. For the structure domain, higher order solid elements with 20 nodes exhibiting quadratic displacement behavior were mainly used. Each node has three degrees of freedom corresponding to the translations in the nodal directions. Only at the front circular body of the plugs 10 node solid elements were used instead, as can be seen on the left of Fig. 7.

A full modal analysis was performed with the unsymmetric method to extract a maximum of 20 modes in the range from 10 to $2000 \mathrm{~Hz}$.

\section{Results}

\subsection{Natural frequencies}

When calculating the natural frequencies, the mass of the caps should be considered. Based on Erturk and Inman (2007) and Shi et al. (2015), who give general expressions for the frequency parameters depending on the tip mass to beam mass ratio (correcting the historical one shown in Blevins (1979)), the theoretical natural frequencies for the empty pipes are shown in Table 5.

The natural frequencies obtained from the physical tests and numerical models are presented in Tables 6 and 7 for the DN32 and DN50 pipes, respectively, together with the percent deviations of the three first modes of vibration for the various filling conditions. Regarding the experimental results, the figures indicate the mean value of the frequency peaks detected from 10 impact tests among the various sensors. The standard deviation for all the values never exceeded $10 \%$ of the mean value. It should be mentioned that
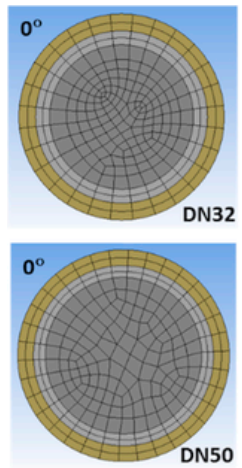
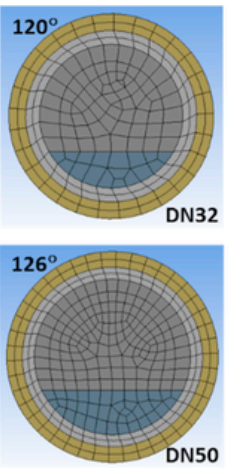
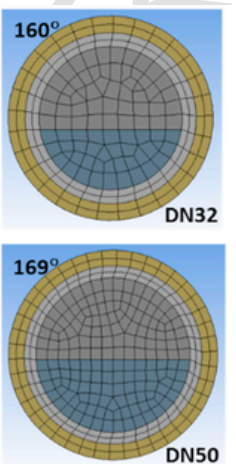
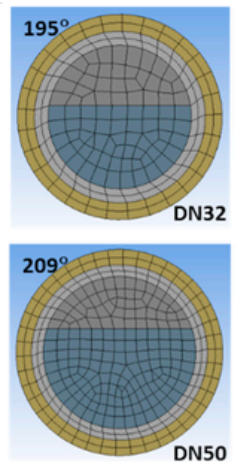
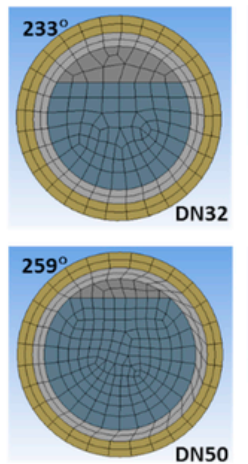
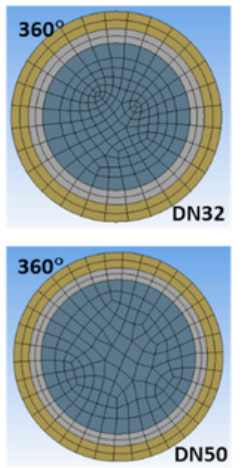

Fig. 8. Cross section of all the meshes for the DN32 and DN50 pipes. The degrees indicate $\theta$ in Fig. 1.

Table 5

Theoretical natural frequencies for $1 \mathrm{~m}$ length empty pipes DN32 and DN50.

\begin{tabular}{lll}
\hline Frequency $[\mathrm{Hz}]$ & DN32 & DN50 \\
\hline$f_{1}$ & 198.0 & 286.7 \\
$f_{2}$ & 562.6 & 814.5 \\
$f_{3}$ & 1128.9 & 1634.3 \\
\hline
\end{tabular}


Table 6

Natural frequencies in $\mathrm{Hz}$ for pipe DN32 obtained from physical tests [Exp] and numerical models [Sim] with percent deviations [Dev] for $f_{1}, f_{2}$ and $f_{3}$.

\begin{tabular}{|c|c|c|c|c|c|c|c|c|}
\hline & & & $h / 2 R$ & & & & & \\
\hline Mode & Axis & DN32 & 0.000 & 0.248 & 0.411 & 0.563 & 0.723 & 1.000 \\
\hline \multirow[t]{3}{*}{$f_{1}$} & \multirow[t]{3}{*}{$\mathrm{Z}$} & $\operatorname{Exp}[\mathrm{Hz}]$ & 200.9 & 196.1 & 192.9 & 188.5 & 185.1 & 181.0 \\
\hline & & $\operatorname{Sim}[\mathrm{Hz}]$ & 199.4 & 195.1 & 191.2 & 187.3 & 183.7 & 179.8 \\
\hline & & $\operatorname{Dev}[\%]$ & -1 & -1 & -1 & -1 & -1 & -1 \\
\hline \multirow[t]{3}{*}{$f_{1}$} & \multirow[t]{3}{*}{$X$} & $\operatorname{Exp}[\mathrm{Hz}]$ & 200.6 & 198.2 & 196.0 & 190.1 & 187.5 & 181.5 \\
\hline & & $\operatorname{Sim}[\mathrm{Hz}]$ & 199.4 & 198.6 & 196.5 & 193.5 & 189.2 & 179.8 \\
\hline & & $\operatorname{Dev}[\%]$ & -1 & 0 & 0 & 2 & 1 & -1 \\
\hline \multirow[t]{3}{*}{$f_{2}$} & \multirow[t]{3}{*}{$\mathrm{Z}$} & $\operatorname{Exp}[\mathrm{Hz}]$ & 555.0 & 542.7 & 531.4 & 521.1 & 510.0 & 497.0 \\
\hline & & $\operatorname{Sim}[\mathrm{Hz}]$ & 556.4 & 543.2 & 531.1 & 520.0 & 509.8 & 497.9 \\
\hline & & $\operatorname{Dev}[\%]$ & 0 & 0 & 0 & 0 & 0 & 0 \\
\hline \multirow[t]{3}{*}{$f_{2}$} & \multirow[t]{3}{*}{$\mathrm{X}$} & $\operatorname{Exp}[\mathrm{Hz}]$ & 556.2 & 546.5 & 538.6 & 525.5 & 514.3 & 499.3 \\
\hline & & $\operatorname{Sim}[\mathrm{Hz}]$ & 556.4 & 553.7 & 547.6 & 538.4 & 525.6 & 497.9 \\
\hline & & $\operatorname{Dev}[\%]$ & 0 & 1 & 2 & 2 & 2 & 0 \\
\hline \multirow[t]{3}{*}{$f_{3}$} & \multirow[t]{3}{*}{$\mathrm{Z}$} & $\operatorname{Exp}[\mathrm{Hz}]$ & 1067.5 & 1049.8 & 1028.8 & 1008.9 & 980.9 & 956.4 \\
\hline & & Sim $[\mathrm{Hz}]$ & 1086.6 & 1059.3 & 1035.3 & 1013.0 & 992.6 & 968.6 \\
\hline & & $\operatorname{Dev}[\%]$ & 2 & 1 & 1 & 0 & 1 & 1 \\
\hline \multirow[t]{3}{*}{$f_{3}$} & \multirow[t]{3}{*}{$\mathrm{X}$} & $\operatorname{Exp}[\mathrm{Hz}]$ & 1077.0 & 1071.5 & 1053.3 & 1020.8 & 1003.7 & 962.1 \\
\hline & & $\operatorname{Sim}[\mathrm{Hz}]$ & 1086.6 & 1081.1 & 1068.7 & 1050.0 & 1024.1 & 968.6 \\
\hline & & $\operatorname{Dev}[\%]$ & 1 & 1 & 1 & 3 & 2 & 1 \\
\hline
\end{tabular}

Table 7

Natural frequencies in Hz for pipe DN50 obtained from physical tests [Exp] and numerical models [Sim] with percent deviations [Dev] for $f_{1}, f_{2}$ and $f_{3}$.

\begin{tabular}{|c|c|c|c|c|c|c|c|c|}
\hline & & & $h / 2 R$ & & & & & \\
\hline Mode & Axis & DN50 & 0.000 & 0.271 & 0.450 & 0.623 & 0.816 & 1.000 \\
\hline \multirow[t]{3}{*}{$f_{1}$} & \multirow[t]{3}{*}{$\mathrm{Z}$} & $\operatorname{Exp}[\mathrm{Hz}]$ & 281.6 & 274.0 & 264.7 & 257.9 & 249.3 & 247.0 \\
\hline & & $\operatorname{Sim}[\mathrm{Hz}]$ & 260.1 & 252.4 & 245.5 & 239.2 & 233.7 & 230.2 \\
\hline & & $\operatorname{Dev}[\%]$ & -8 & -8 & -7 & -7 & -6 & -7 \\
\hline \multirow[t]{3}{*}{$f_{1}$} & \multirow[t]{3}{*}{$\mathrm{X}$} & $\operatorname{Exp}[\mathrm{Hz}]$ & 283.0 & 277.7 & 276.0 & 260.7 & 251.5 & 247.8 \\
\hline & & $\operatorname{Sim}[\mathrm{Hz}]$ & 260.1 & 258.4 & 254.5 & 248.3 & 239.8 & 230.2 \\
\hline & & $\operatorname{Dev}[\%]$ & -8 & -7 & -8 & -5 & -5 & -7 \\
\hline \multirow[t]{3}{*}{$f_{2}$} & \multirow[t]{3}{*}{$\mathrm{Z}$} & $\operatorname{Exp}[\mathrm{Hz}]$ & 762.2 & 739.0 & 713.0 & 696.0 & 673.1 & 670.0 \\
\hline & & $\operatorname{Sim}[\mathrm{Hz}]$ & 728.9 & 704.3 & 682.9 & 663.6 & 647.4 & 636.3 \\
\hline & & $\operatorname{Dev}[\%]$ & -4 & -5 & -4 & -5 & -4 & -5 \\
\hline \multirow[t]{3}{*}{$f_{2}$} & \multirow[t]{3}{*}{$\mathrm{X}$} & $\operatorname{Exp}[\mathrm{Hz}]$ & 763.2 & 742.8 & 740.3 & 698.5 & 684.9 & 673.0 \\
\hline & & $\operatorname{Sim}[\mathrm{Hz}]$ & 728.9 & 723.3 & 710.8 & 691.4 & 665.0 & 636.3 \\
\hline & & $\operatorname{Dev}[\%]$ & -4 & -3 & -4 & -1 & -3 & -5 \\
\hline \multirow[t]{3}{*}{$f_{3}$} & \multirow[t]{3}{*}{$\mathrm{Z}$} & $\operatorname{Exp}[\mathrm{Hz}]$ & 1314.4 & 1284.5 & 1246.3 & 1213.8 & 1172.8 & 1168.3 \\
\hline & & $\operatorname{Sim}[\mathrm{Hz}]$ & 1409.3 & 1358.7 & 1315.2 & 1277.0 & 1245.8 & 1222.9 \\
\hline & & $\operatorname{Dev}[\%]$ & 7 & 6 & 6 & 5 & 6 & 5 \\
\hline \multirow[t]{3}{*}{$f_{3}$} & \multirow[t]{3}{*}{$\mathrm{X}$} & $\operatorname{Exp}[\mathrm{Hz}]$ & 1331.3 & 1311.2 & 1294.7 & 1248.2 & 1195.1 & 1179.5 \\
\hline & & $\operatorname{Sim}[\mathrm{Hz}]$ & 1409.3 & 1397.8 & 1371.5 & 1331.5 & 1278.9 & 1222.9 \\
\hline & & Dev [\%] & 6 & 7 & 6 & 7 & 7 & 4 \\
\hline
\end{tabular}

since both the sensor locations and the expected maximum deflections for all the modes are well known, those sensors close to a node for a particular mode shape have not been taken into account for the standard deviation calculation.

For the numerical results for pipe DN32, the maximum percent deviation for equivalent values measured in the physical tests is $2 \%$. For pipe DN50, the maximum percent deviation is $8 \%$. The differences between the values from the simulations and physical tests are explained firstly by the uncertainty of both sets of data. Secondly, the assumed model simplifications in terms of ideal material properties, fluid behavior, geometry and boundary conditions are also error sources. As observed in the numerical results, both horizontal and vertical natural frequencies are equal when the pipes are empty or completely full of water. The experimental results instead show slightly higher lateral frequencies for those cases; this discrepancy is due to the simplification of the model, assuming completely cylindrical caps results in a complete symmetrical geometry in both considered axes of vibration.

\subsection{Frequency ratios}

As observed in Tables 6 and 7, the natural frequencies of the pipe are maximum when empty, $f_{a}$, and decrease as the water level increases until the minimum value is found for the fully filled case, $f_{w}$. The reduction of natural frequencies due to the added mass effect is mainly a result of the inertia of the surrounding fluid entrained by the accelerating structure.

Zhang et al. (1999) found that the natural frequencies of a water-filled pipe were about $15 \%$ lower than those of an air-filled pipe due to the extra mass of the water according to the frequency ratio given in Eq. (13). 
$\frac{f_{w}}{f_{a}}=\sqrt{\frac{\rho_{s} A_{s}+\rho_{a} A_{f}}{\rho_{s} A_{s}+\rho_{f} A_{f}}}$

where $\rho$ is mass density, $A$ is cross-sectional area, and the subscripts $a, f$ and $s$ stand for air, fluid and structure, respectively. Table 8 presents the water to air frequency ratios found with the dead mass approach (Zhang et al., 1999) applied to partly filled DN32 and DN50 pipes.

The frequency ratios found with the experiments are indicated in Tables 9 and 10 for DN32 and DN50, respectively, as well as the corresponding percent deviations of Zhang's predictions and of numerical simulations.

Table 8

Water to air frequency ratios for pipes DN32 and DN50 obtained from Eq. (13).

\begin{tabular}{lllllll}
\hline \multirow{2}{*}{ DN32 } & $h / 2 R$ & 0.000 & 0.248 & 0.411 & 0.563 & 0.723 \\
& Eq. (13) & 1,00 & 0,97 & 0,94 & 0,92 & 0,89 \\
DN50 & $h / 2 R$ & 0.000 & 0.271 & 0.450 & 0.623 & 0.816 \\
& Eq. (13) & 1,00 & 0,93 & 0,87 & 0,83 & 0,79 \\
\hline
\end{tabular}

Table 9

Experimental frequency ratios for pipe DN32 with percent deviations of Zhang's predictions [Zhang] and simulation results [Sim] for $f_{1}, f_{2}$ and $f_{3}$.

\begin{tabular}{|c|c|c|c|c|c|c|c|c|}
\hline & & & $h / 2 R$ & & & & & \\
\hline Mode & Axis & DN32 & 0.000 & 0.248 & 0.411 & 0.563 & 0.723 & 1.000 \\
\hline \multirow[t]{3}{*}{$f_{1}$} & \multirow[t]{3}{*}{$\mathrm{Z}$} & $\operatorname{Exp}[\mathrm{Hz}]$ & 1,00 & 0,98 & 0,96 & 0,94 & 0,92 & 0,90 \\
\hline & & Zhang [\%] & 0 & -1 & -2 & -2 & -3 & -3 \\
\hline & & $\operatorname{Sim}[\%]$ & 0 & 0 & 0 & 0 & 0 & 0 \\
\hline \multirow[t]{3}{*}{$f_{1}$} & \multirow[t]{3}{*}{$\mathrm{X}$} & $\operatorname{Exp}[\mathrm{Hz}]$ & 1,00 & 0,99 & 0,98 & 0,95 & 0,93 & 0,90 \\
\hline & & Zhang [\%] & 0 & -2 & -3 & -3 & -4 & -4 \\
\hline & & $\operatorname{Sim}[\%]$ & 0 & 1 & 1 & 2 & 2 & 0 \\
\hline \multirow[t]{3}{*}{$f_{2}$} & \multirow[t]{3}{*}{$\mathrm{Z}$} & $\operatorname{Exp}[\mathrm{Hz}]$ & 1,00 & 0,98 & 0,96 & 0,94 & 0,92 & 0,90 \\
\hline & & Zhang [\%] & 0 & -1 & -2 & -2 & -3 & -3 \\
\hline & & $\operatorname{Sim}[\%]$ & 0 & 0 & 0 & 0 & 0 & 0 \\
\hline \multirow[t]{3}{*}{$f_{2}$} & \multirow[t]{3}{*}{$\mathrm{X}$} & $\operatorname{Exp}[\mathrm{Hz}]$ & 1,00 & 0,98 & 0,97 & 0,94 & 0,92 & 0,90 \\
\hline & & Zhang [\%] & 0 & -1 & -3 & -3 & -3 & -3 \\
\hline & & $\operatorname{Sim}[\%]$ & 0 & 1 & 2 & 2 & 2 & 0 \\
\hline \multirow[t]{3}{*}{$f_{3}$} & \multirow[t]{3}{*}{$\mathrm{Z}$} & $\operatorname{Exp}[\mathrm{Hz}]$ & 1,00 & 0,98 & 0,96 & 0,95 & 0,92 & 0,90 \\
\hline & & Zhang [\%] & 0 & -1 & -2 & -3 & -3 & -3 \\
\hline & & $\operatorname{Sim}[\%]$ & 0 & -1 & -1 & -1 & -1 & -1 \\
\hline \multirow[t]{3}{*}{$f_{3}$} & \multirow[t]{3}{*}{$\mathrm{X}$} & $\operatorname{Exp}[\mathrm{Hz}]$ & 1,00 & 0,99 & 0,98 & 0,95 & 0,93 & 0,89 \\
\hline & & Zhang [\%] & 0 & -2 & -4 & -3 & -4 & -3 \\
\hline & & $\operatorname{Sim}[\%]$ & 0 & 0 & 1 & 2 & 1 & 0 \\
\hline
\end{tabular}

Table 10

Experimental frequency ratios for pipe DN50 with percent deviations of Zhang's predictions [Zhang] and simulation results [Sim] for $f_{1}, f_{2}$ and $f_{3}$.

\begin{tabular}{|c|c|c|c|c|c|c|c|c|}
\hline & & & $h / 2 R$ & & & & & \\
\hline Mode & Axis & DN50 & 0.000 & 0.271 & 0.450 & 0.623 & 0.816 & 1.000 \\
\hline \multirow[t]{3}{*}{$f_{1}$} & \multirow[t]{3}{*}{$\mathrm{Z}$} & $\operatorname{Exp}[\mathrm{Hz}]$ & 1,00 & 0,97 & 0,94 & 0,92 & 0,89 & 0,88 \\
\hline & & Zhang [\%] & 0 & -4 & -7 & -10 & -11 & -13 \\
\hline & & $\operatorname{Sim}[\%]$ & 0 & 0 & 0 & 0 & 1 & 1 \\
\hline \multirow[t]{3}{*}{$f_{1}$} & \multirow[t]{3}{*}{$\mathrm{X}$} & $\operatorname{Exp}[\mathrm{Hz}]$ & 1,00 & 0,98 & 0,98 & 0,92 & 0,89 & 0,88 \\
\hline & & Zhang [\%] & 0 & -5 & -10 & -10 & -11 & -12 \\
\hline & & $\operatorname{Sim}[\%]$ & 0 & 1 & 0 & 4 & 4 & 1 \\
\hline \multirow[t]{3}{*}{$f_{2}$} & \multirow[t]{3}{*}{$\mathrm{Z}$} & $\operatorname{Exp}[\mathrm{Hz}]$ & 1,00 & 0,97 & 0,94 & 0,91 & 0,88 & 0,88 \\
\hline & & Zhang [\%] & 0 & -4 & -6 & -9 & -11 & -13 \\
\hline & & $\operatorname{Sim}[\%]$ & 0 & 0 & 0 & 0 & 1 & -1 \\
\hline \multirow[t]{3}{*}{$f_{2}$} & \multirow[t]{3}{*}{$\mathrm{X}$} & $\operatorname{Exp}[\mathrm{Hz}]$ & 1,00 & 0,97 & 0,97 & 0,92 & 0,90 & 0,88 \\
\hline & & Zhang [\%] & 0 & -4 & -10 & -10 & -12 & -13 \\
\hline & & $\operatorname{Sim}[\%]$ & 0 & 2 & 1 & 4 & 2 & -1 \\
\hline \multirow[t]{3}{*}{$f_{3}$} & \multirow[t]{3}{*}{$\mathrm{Z}$} & $\operatorname{Exp}[\mathrm{Hz}]$ & 1,00 & 0,98 & 0,95 & 0,92 & 0,89 & 0,89 \\
\hline & & Zhang [\%] & 0 & -5 & -8 & -10 & -12 & -14 \\
\hline & & $\operatorname{Sim}[\%]$ & 0 & -1 & -2 & -2 & -1 & -2 \\
\hline \multirow[t]{3}{*}{$f_{3}$} & \multirow[t]{3}{*}{$\mathrm{X}$} & $\operatorname{Exp}[\mathrm{Hz}]$ & 1,00 & 0,98 & 0,97 & 0,94 & 0,90 & 0,89 \\
\hline & & Zhang [\%] & 0 & -5 & -10 & -12 & -12 & -13 \\
\hline & & $\operatorname{Sim}[\%]$ & 0 & 1 & 0 & 1 & 1 & -2 \\
\hline
\end{tabular}


In all the cases, the deviations of the numerical results are lower than Zhang's deviations. The maximum deviations of the numerical results are found for the larger pipe DN50 with values up to 4\%. Meanwhile, the Zhang's maximum ones are up to $14 \%$ for the same pipe size. It can also be noted that deviations are larger in horizontal direction than in vertical direction.

These observations point out that the numerical method permits to take into account the influence of the mode shape on the added mass effect which is not taken into account by Zhang's ratio. Moreover, the numerical method permits to capture the asymmetry effects of the coupled solid-fluid system when the pipe is partially filled. In this case, the better performance of the numerical approach is because Zhang's equation was developed for fully filled pipes; although its range of application has been extended here for comparison purposes only.

\subsection{Added mass coefficients}

The fundamental frequency of a given mode for a lightly damped structure vibrating in air, $f_{a}$, can be expressed with Eq. (14):

$$
f_{a}=\sqrt{\frac{k}{m}}
$$

where $k$ and $m$ are the modal stiffness and the modal mass respectively. Whereas when submerged in water, the dynamic response of the solid body is altered by the effect of the water added mass, $m_{w}$, as given by Eq. (15):

$f_{w}=\sqrt{\frac{k}{m+m_{w}}}$

where $f_{w}$ is the natural frequency in water. Consequently, the added mass coefficient, $C_{M}=m_{w} / m$, can be defined based on the ratio between $f_{a}$ and $f_{w}$ with Eq. (16):

$C_{M}=\left(\frac{f_{a}}{f_{w}}\right)^{2}-1$

The added mass coefficients, $C_{M}$, for both pipes are plotted in Fig. 9 as a function of $h / 2 R$. As it can be seen, the added mass effects increase with the volume of water filling the pipe and the maximum values are different depending on the pipe size. For DN32, they reach values around 0.25 , meanwhile for DN50 they reach values of approximately 0.3 . Vertical and horizontal values for a given height of water separate because they follow different trends. In all partially filled conditions, vertical effects are slightly higher than the horizontal ones. This behavior is analogous for both pipe sizes. For the fully filled pipe condition, values in both di-
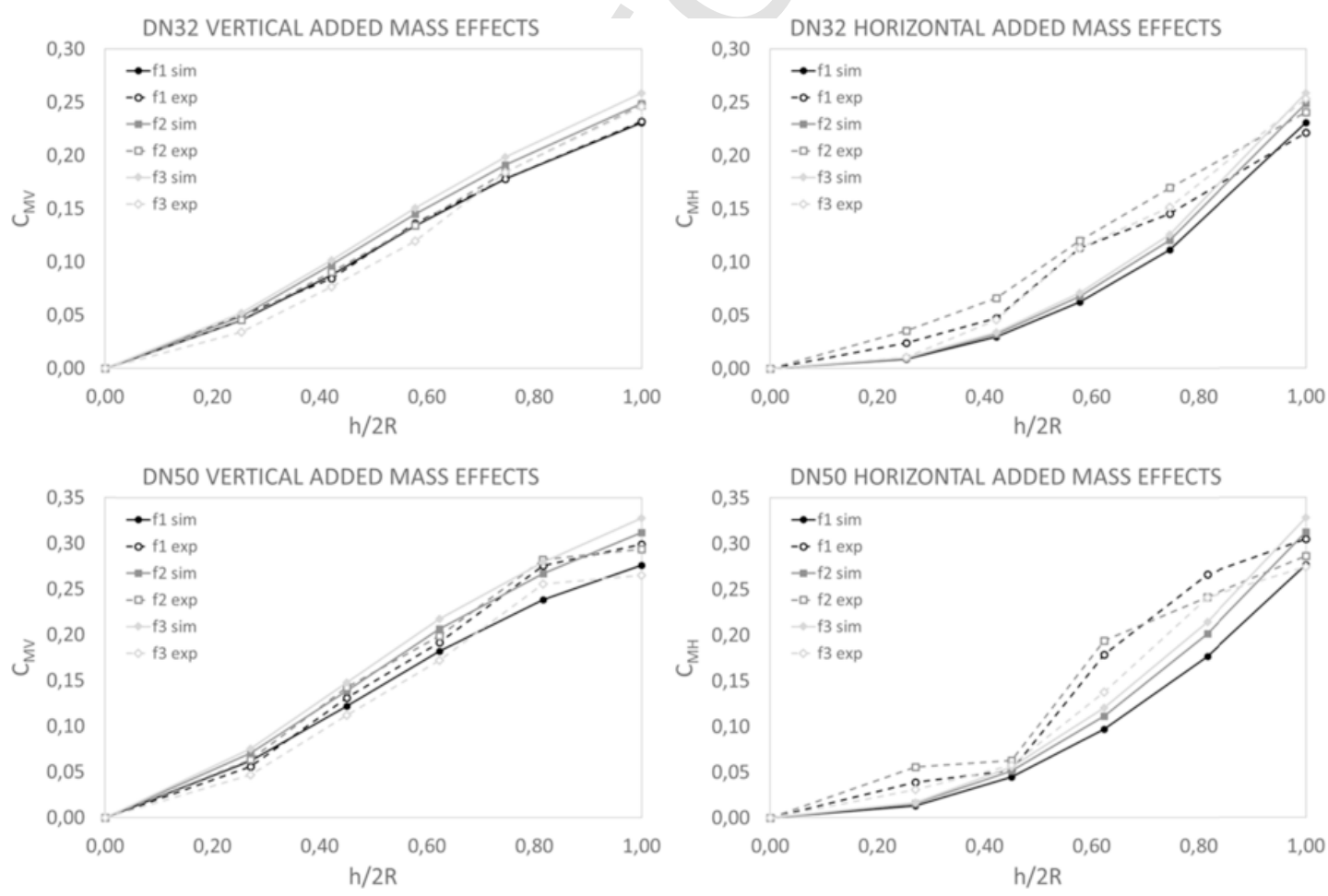

Fig. 9. Vertical (left) and horizontal (right) added mass coefficients of $f_{1}, f_{2}$ and $f_{3}$ as a function of $\mathrm{h} / 2 \mathrm{R}$ for DN32 (top) and DN50 (bottom) pipes. 
rections are close to each other. In general, the largest deviations between numerical and experimental results are observed in lateral direction as expected when building the simplified model.

\section{Added mass estimators}

A theoretical added mass estimator, $A M$, has been defined based on the static forces of the filling water applied to the wetted inner pipe walls. The vertical and horizontal water forces, $F_{w V}$ and $F_{w H}$, can be calculated with Eqs. (17) and (18), respectively.

$F_{w V}=\rho_{w} g V_{w}=\rho_{w} g L A_{w}=\rho_{w} g L \frac{R^{2}}{2}(\theta-\sin \theta)$

$F_{w H}=\rho_{w} g \frac{h}{2} A_{w H}=\rho_{w} g \frac{h}{2} L h=\rho_{w} g \frac{L}{2} R^{2}\left(1-\cos \frac{\theta}{2}\right)^{2}$

Where $\rho_{w}$ is the water density and $A_{w H}$ is the lateral projection of the circular segment. From them, the vertical and the horizontal added mass estimators, $A M_{V}$ and $A M_{H}$, can be calculated with Eqs. (19) and (20), respectively, using the mass of the pipe, $m$.

$A M_{V}=\frac{F_{w V} / g}{m}=\frac{\rho_{w} L \frac{R^{2}}{2}(\theta-\sin \theta)}{\rho \pi e(e+2 R) L}=\frac{\rho_{w} R^{2}}{\rho 2 \pi e(e+2 R)}(\theta-\sin \theta)$

$A M_{H}=\frac{F_{w H} / g}{m}=\frac{\rho_{w} \frac{L}{2} R^{2}\left(1-\cos \frac{\theta}{2}\right)^{2}}{\rho \pi e(e+2 R) L}=\frac{\rho_{w} R^{2}}{\rho 2 \pi e(e+2 R)}\left(1-\cos \frac{\theta}{2}\right)^{2}$

In both expressions a constant, $a$, that takes into account the pipe geometry and the liquid and solid densities, is found as indicated in Eq. (21).

$a=\frac{\rho_{w} R^{2}}{\rho 2 \pi e(e+2 R)}$

As a result, for any given pipe and liquid, $A M_{V}$ and $A M_{H}$ can be estimated with Eqs. (22) and (23), respectively.

$A M_{V}=a(\theta-\sin \theta)$

$A M_{H}=a\left(1-\cos \frac{\theta}{2}\right)^{2}$

If the vertical and horizontal added mass coefficients obtained experimentally, $C_{M V}$ and $C_{M H}$, are plotted as a function of Eqs. (22) and (23), respectively, a first degree and a second degree polynomial trends are found independently of the pipe cross section dimensions, as can be observed in Fig. 10. The equations that best fits the current results for both DN32 and DN50 can be expressed as (24) and (25):
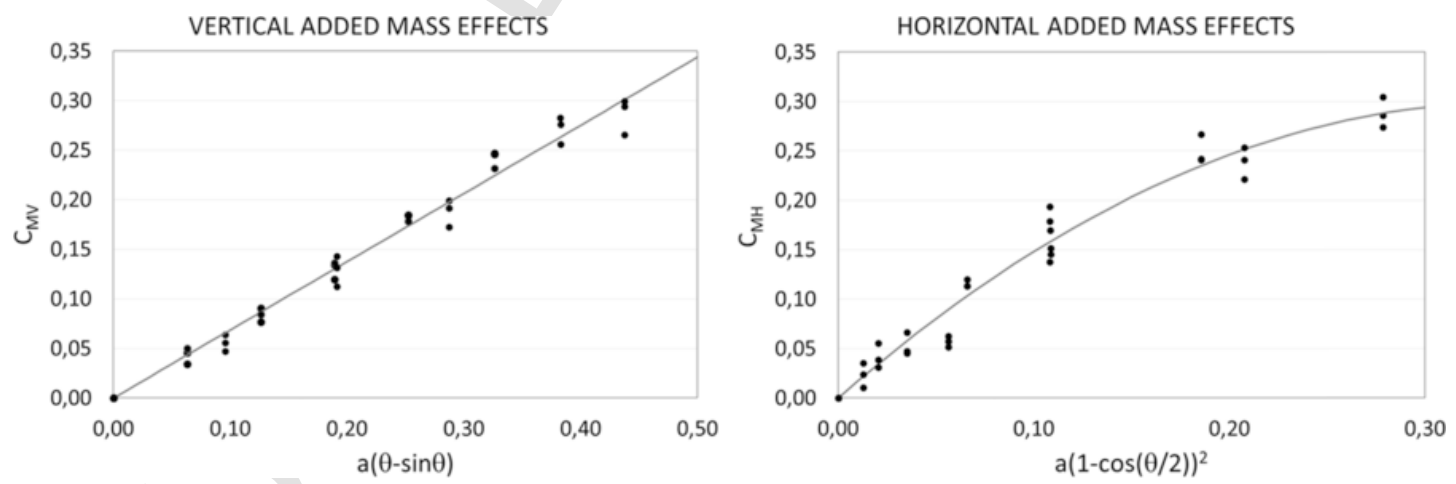

Fig. 10. Experimental vertical (left) and horizontal (right) added mass coefficients of $f_{1}, f_{2}$ and $f_{3}$ for both DN32 and DN50 pipes as a function of Eqs. (22) and (23), respectively, and corresponding curve fittings indicated in Eqs. (24) and (25) based on the estimators. 


$$
\begin{aligned}
& C_{M V}=0.688 A M_{V} \\
& C_{M H}=-2.503 A M_{H}^{2}+1.731 A M_{H}
\end{aligned}
$$

The correlation coefficients $R^{2}$ of both curve fittings are approximately $98 \%$ and $97 \%$ for vertical and horizontal data respectively.

\section{Potential application}

From a practical point of view, the added mass estimators are expected to be applicable to any other horizontally mounted cylindrical pipe with a different size. Based on them, it would be possible to estimate the expected frequency ratios without the need for time consuming experiment or simulation. With this information, we propose to predict air content in industrial conditions with the simple use of an external accelerometer and a frequency analyzer. The method would consist of exciting the pipe with a hammer and measuring the induced vibrations when the pipe is under nominal operating conditions and completely filled with water. Then, regular periodic repetitions of the tests would permit to track if the natural frequencies of the pipe are increasing. In that case, the increasing rate compared with the estimators would serve to decide if a significant amount of air might be responsible of such variations and if maintenance action on the piping system like purging is required.

It is important to highlight here that the strength of the present approach is the simplification in terms of calculations required to obtain a valid result. However, it is evident that neglecting all fluid dynamics effects happening within the pipe can only be done under certain circumstances. In fact, the results in Tables 6 and 7 show that the error increases between DN32 and DN50 for the whole set of frequencies. The relative importance of sloshing and other fluid effects are expected to increase with the diameter. Therefore, this method would eventually have a range of application based on the diameter of the pipe and which would define the accuracy level.

\section{Conclusions}

The three first vertical and horizontal modes of vibration for free horizontal pipes have been experimentally and numerically obtained for various filling ratios ranging from empty to completely water filled cases. The numerical results for two different nominal diameter pipes show a good level of agreement with maximum deviations of about $8 \%$ in natural frequencies. The vertical and horizontal added mass effects present different behaviors as a function of filling ratio. Moreover, the pipe cross section dimensions also have an influence on the added mass effects. Two new added mass estimators are proposed that permit to infer the vertical and horizontal effects for any filling condition and pipe dimensions. These results can be used for the detection of air content in pipes.

\section{Acknowledgements}

The second and third author would like to acknowledge the support of the Science Foundation Ireland through the National Centre for Marine and Renewable Energy Ireland (MaREI) (Grant no. 12/RC/2302). The third author would also like to acknowledge the support of Science Foundation Ireland through the Career Development Award programme (Grant no. 13/CDA/2200).

\section{References}

Amabili, M., 1996. Free vibration of partially filled, horizontal cylindrical shells. J. Sound Vib. 191 (5), 757-780.

Amabili, M., 1997. Shell-plate interaction in the free vibrations of circular cylindrical tanks partially filled with a liquid: the artificial spring method. J. Sound Vib. 199 (3), 431-452.

Amabili, M., 1997. Flexural vibration of cylindrical shells partially coupled with external and internal fluids. J. Vib. Acoust. 119, $476-484$.

Amabili, M., 1999. Vibrations of circular tubes and shells filled and partially immersed in dense fluids. J. Sound Vib. 221 (4), 567-585.

Amabili, M., Dalpiaz, G., 1995. Breathing vibrations of a horizontal circular cylindrical tank shell, partially filled with liquid. ASME J. Vib. Acoust. 117 (2), 187-191.

Amabili, M., Pangnanelli, F., Pellegrini, M., 2001. Experimental modal analysis of a water-filled circular cylindrical shell. Trans. Built Environ. 56.

Blevins, R., 1979. Formulas for Natural Frequency and Mode Shape. Krieger Publishing Company, Florida.

Chen, W., Ding, H., 1999. Natural frequencies of fluid-filled transversely isotropic cylindrical shells. Int. J. Mech. Sci. 41 (6), $677-684$.

Chiba, M., Yamaki, N., Tani, J., 1984. Free vibration of a clamped-free circular cylindrical shell partially filled with liquid-Part I: theoretical analysis. Thin-Walled Struct. 2 (3), 265-284.

Chiba, M., Yamaki, N., Tani, J., 1984. Free vibration of a clamped-free circular cylindrical shell partially filled with liquid-Part II: numerical results. Thin-Walled Struct. 2 (4), 307-324.

Chiba, M., Yamaki, N., Tani, J., 1985. Free vibration of a clamped-free circular cylindrical shell partially filled with liquid-Part III: experimental results. Thin-Walled Struct. 3 (1), 1-14.

Ergin, A., Temarel, P., 2002. Free vibration of a partially liquid-filled and submerged, horizontal cylindrical shell. J. Sound Vib. 254 (5), $951-965$.

Erturk, A., Inman, D.J., 2007. On the fundamental transverse vibration frequency of a free-free thin beam with identical end masses. J. Vib. Acoust. 129 (5), 656-662. Escarameia, M., 2007. Investigating hydraulic removal of air from water pipelines. Proc. Inst. Civil Eng.: Water Manag. 160 (1), 25-34.

Govier, G.W., Omer, M.M., 1962. The horizontal pipeline flow of air-water mixtures. Can. J. Chem. Eng. 40 (3), $93-104$.

Lavooij, C.S.W, Tijsseling, A.S., 1991. Fluid-structure interaction in liquid-filled piping systems. J. Fluids Struct. 5 (5), $573-595$.

Li, S., Karney, B.W., Liu, G., 2015. FSI research in pipeline systems - a review of the literature. J. Fluids Struct. 57, $277-297$.

Lubbers, C., Clemens, F., 2007. Detection of gas pockets in pressurised waste water mains using dynamic system response analysis. Water Sci. Technol. 55 (4), 31-38.

Mazúch, T., Horacek, J., Trnka, J., Veselý, J., 1996. Natural modes and frequencies of a thin clamped-free steel cylindrical storage tank partially filled with water: fem and measurement. J. Sound Vib. 193 (3), 669-690. 
Warburton, G.N., 1965. Vibration of thin cylindrical shells. J. Mech. Eng. Sci. 7 (4).

Zhang, L., Tijsseling, A.S., Vardy, E., 1999. FSI analysis of liquid-filled pipes. J. Sound Vib. 224 (1), 69-99. 